\title{
$\beta$-Elemene Triggers ROS-dependent Apoptosis in Glioblastoma Cells Through Suppressing STAT3 Signaling Pathway
}

\author{
Shi-zhong Cai ${ }^{1 \dagger}$, Qian-wei Xiong ${ }^{2 \dagger}$, Li-na Zhao ${ }^{3}, Y_{i-t i n g ~} \mathrm{Ji}^{1}$, Zheng-xiang Luo ${ }^{4 *}$ and \\ Zhou-rui Ma ${ }^{2 *}$
}

${ }^{1}$ Department of Child and Adolescent Healthcare, Children's Hospital of Soochow University, Suzhou, China, ${ }^{2}$ Department of Surgery, Children's Hospital of Soochow University, Suzhou, China, ${ }^{3}$ Department of Laboratory Medicine, Key Laboratory of Clinical Immunology of Jiangsu Province, The First Affiliated Hospital of Soochow University, Suzhou, China, ${ }^{4}$ Department of Neurosurgery, Nanjing Brain Hospital Affiliated to Nanjing Medical University, Nanjing, China

OPEN ACCESS

Edited by:

Andrea Ladányi,

National Institute of Oncology (NIO),

Hungary

*Correspondence:

Zhou-rui Ma

chngrey@suda.edu.cn

Zheng-xiang Luo

zhengxiang/uo@126.com

${ }^{t}$ These authors contributed equally to this work.

Received: 13 August 2020 Accepted: 15 February 2021

Published: 25 March 2021

Citation:

Cai S, Xiong Q, Zhao L, Ji Y, Luo Z and Ma Z (2021) $\beta$-Elemene Triggers ROSdependent Apoptosis in Glioblastoma Cells Through Suppressing STAT3

Signaling Pathway.

Pathol. Oncol. Res. 27:594299.

doi: 10.3389/pore.2021.594299
Glioblastoma is one of the most aggressive primary brain tumors with few treatment strategies. $\beta$-Elemene is a sesquiterpene known to have broad spectrum antitumor activity against various cancers. However, the signaling pathways involved in $\beta$-elemene induced apoptosis of glioblastoma cells remains poorly understood. In this study, we reported that $\beta$-elemene exhibited antiproliferative activity on U87 and SHG-44 cells, and induced cell death through induction of apoptosis. Incubation of these cells with $\beta$-elemene led to the activation of caspase-3 and generation of reactive oxygen species (ROS). Western blot assay showed that $\beta$-elemene suppressed phosphorylation of STAT3, and subsequently down-regulated the activation of $p$-JAK2 and $p$-Src. Moreover, pre-incubation of cells with ROS inhibitor $N$-acetyl-L-cysteine (NAC) significantly reversed $\beta$-elemene-mediated apoptosis effect and down-regulation of JAK2/Src-STAT3 signaling pathway. Overall, our findings implied that generation of ROS and suppression of STAT3 signaling pathway is critical for the apoptotic activity of $\beta$-elemene in glioblastoma cells.

Keywords: $\beta$-elemene, glioblastoma, apoptosis, stat3, ROS

\section{INTRODUCTION}

Glioblastoma (GBM) is one of the most aggressive malignancies with high recurrence rate and low survival rate [1]. Temozolomide (TMZ) is the first-line chemotherapeutic strategy for the treatment of GBM currently. Although TMZ has been proven to effectively inhibit GBM proliferation, intrinsic and acquired resistance in GBM cells ultimately limits its efficacy [2]. Development of novel chemotherapeutic drugs for GBM is hampered by many difficulties, like drug resistance, toxicity, disability to cross the blood-brain barrier [3]. Thus, research and development of new drugs with promising therapeutic effects and lower toxicity for GBM treatment is urgently needed [4]. Great efforts have been made to understand the basic biology of GBM and many molecular signaling pathways have been identified as potential therapeutic targets for this devastating cancer [5].

Up-regulation and hyperactivation of signal transducer and activator of transcription 3 (STAT3) in GBM have been identified by numerous studies, and aberrant STAT3 activation is associated with poor prognosis [6]. Seven different STATs $(1,2,3,4,5 \mathrm{a}, 5 \mathrm{~b}$, and 6$)$ have been found in mammalian cells until now, and of all the STATs, STAT3 is certainly the most eminent among cancers. It can be 
activated by a variety of stimuli, including cytokines, growth factors and interferons [7]. In a high percentage of GBM cells, persistent activation of STAT3 induces cell proliferation, antiapoptosis, glioma stem cell maintenance, tumor invasion and immune evasion [8]. Thus, STAT3 is a potential therapeutic target of GBM, and many small molecular inhibitors targeting STAT3 have been developed as effective strategies for the treatment of GBM [9].

$\beta$-Elemene, a sesquiterpene, is the main component of elemene, which was extracted from the traditional Chinese medicinal herb Curcuma wenyujin [10]. $\beta$-Elemene is renowned for its anticancer activity against various of cancers by inhibiting cell proliferation, arresting cell cycle, inducing an apoptotic trigger, downregulating anti-apoptotic signals, decreasing mitochondrial potential, and enhancing the activity of the immune system [11]. For example, $\beta$-elemene has been widely studied for the treatment of breast cancer [12], lung cancer [13], brain tumor [14], and other malignant tumors. In addition, a number of novel $\beta$-elemene derivatives exhibiting potent antiglioma activity have been reported by structural modification of $\beta$-elemene [15]. These studies suggest that $\beta$-elemene might be a precious candidate for future anticancer medications to treat GBM.

In this study, we found that $\beta$-elemene inhibited the proliferation of U87 and SHG-44 cells. $\beta$-Elemene triggers ROS-dependent apoptosis in glioblastoma cells through suppressing STAT3 signaling pathway, it suppressed phosphorylation of STAT3, and subsequently down-regulated the activation of $p$-JAK2 and $p$-Src.

\section{MATERIALS AND METHODS}

\section{Cell Lines and Cell Culture}

The glioma cell lines SHG-44 and U87 were purchased from the Type Culture Collection of the Chinese Academy of Sciences (Shanghai, China). All cells were cultured in Dulbecco's modified Eagle's medium (DMEM) supplemented with $10 \%$ fetal bovine serum (FBS), $50 \mathrm{IU} / \mathrm{ml}$ penicillin and $50 \mathrm{mg} / \mathrm{ml}$ streptomycin (Gibco, Grand Island, NY, United States) under the conditions of $37^{\circ} \mathrm{C}$ in a humidified atmosphere with $5 \% \mathrm{CO}_{2}$.

\section{Reagents}

$\beta$-Elemene was purchased from Dalian Jingan Pharmaceutical Co. China (Purity 99.5\%). MTT (3-(4,5)-dimethylthiahiazo (-z-y1)-3,5-di-phenytetrazoliumromide), DCF-DA (2,7dichlorodihydrofluorescein diacetate), NAC (N-Acetyl-Lcysteine) were purchased from Sigma-Aldrich (St. Louis, MO, United States).

\section{Cell Viability Assay}

Cell viability was detected using MTT assay. $5 \times 10^{3}$ cells were seeded into 96-well plates, after incubation for $24 \mathrm{~h}$, the cells were treated with the indicated concentrations of $\beta$-elemene for 24,48 , or $72 \mathrm{~h}$. Then, $10 \mu \mathrm{l} \mathrm{of} 0.5 \mathrm{mg} / \mathrm{ml}$ MTT was added and the mixture was incubated at $37^{\circ} \mathrm{C}$ for another $4 \mathrm{~h}$. The culture medium was removed and
$100 \mu \mathrm{l}$ DMSO was added to dissolve the formazan crystals. The absorbance at $490 \mathrm{~nm}$ was measured using a microplate reader (Bio-Tek, United States).

\section{Caspase-3 Activity Measurement}

The activity of caspase- 3 was detected using the Caspase- 3 Activity Assay Kit (Beyotime, Jiangsu, China) according to the manufacturer's recommendation. The absorbance at $405 \mathrm{~nm}$ was measured using a microplate reader after treatment with $\beta$-elemene for $12 \mathrm{~h}$ (Bio-Tek, Vermont, United States).

\section{Detection of Intracellular ROS}

ROS levels were detected using probe 2,7dichlorodihydrofluorescein diacetate (DCF-DA). In brief, cells were treated with different concentrations of $\beta$-elemene for $12 \mathrm{~h}$ with or without pre-incubation of $5 \mathrm{mM} \mathrm{NAC} \mathrm{for} 30 \mathrm{~min}$, then incubated with $10 \mu \mathrm{M}$ DCF-DA for $20 \mathrm{~min}$ at $37^{\circ} \mathrm{C}$. PBS was used to wash the cells for three times before detection. Fluorescence microscope (Olympus, Tokyo, Japan) was used for direct observation. To detect fluorescence intensity, cells were collected and fluorescence was detected with a flow cytometry (FACScan, BD Biosciences, United States).

\section{Annexin V-FITC/PI Assay}

Annexin V-FITC/PI apoptosis detection kit was used to perform the apoptotic assay following the manufacturer's instructions (Beyotime, Jiangsu, China). Briefly, after treatment, $1 \times 10^{6}$ cells were washed using $1 \mathrm{ml}$ binding buffer for three times, then centrifuged at $300 \times \mathrm{g}$ and stained with $10 \mu \mathrm{l}$ Annexin V-FITC solution at $37^{\circ} \mathrm{C}$ for $15 \mathrm{~min}$. Before detecting, $5 \mu \mathrm{l}$ PI solution was added to the samples and the apoptotic cells were detected using flow cytometry (BD Biosciences, United States).

\section{Western Blot Analysis}

Cells after treatment were lysed and centrifuged at $12,000 \times g$ for $15 \mathrm{~min}$. The supernatant was collected and the protein concentrations were detected using the BCA assay kit (Beyotime, Jiangsu, China). Equal amounts of total proteins $(20-60 \mu \mathrm{g})$ were separated using SDS-polyacrylamide gel and electrotransferred to PVDF membranes. The membranes were initially blocked with $5 \%$ nonfat dry milk in PBS-Tween-20 $(0.1 \%, \mathrm{v} / \mathrm{v})$ at $4^{\circ} \mathrm{C}$ for $12 \mathrm{~h}$ and then probed with primary antibodies against cleaved caspase-3 (mouse IgG1), Bcl-2

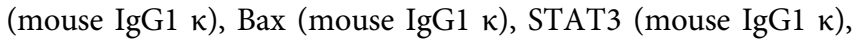
p-STAT3 (mouse IgG1), p-JAK2 (Rabbit mAb), JAK2 (mouse IgG2b $\kappa$ ), $p$-Src (mouse IgG2a $\kappa$ ), Src (mouse IgG2a $\kappa$ ) or GAPDH (Rabbit mAb) (Santa Cruz, CA, United States), which were diluted following the manufacturer's instructions at $4^{\circ} \mathrm{C}$ overnight. Then the membranes were blotted with the horseradish peroxidase-conjugated anti-mouse or anti-rabbit secondary antibodies (Beyotime, Jiangsu, China, 1:5,000) for $2 \mathrm{~h}$ at $37^{\circ} \mathrm{C}$. Finally, the bands were visualized through the enhanced chemiluminescence protocol. Signals were densitometrically quantified and normalized to GAPDH. The results were analyzed using Image J software $(\mathrm{NIH}$, United States). 

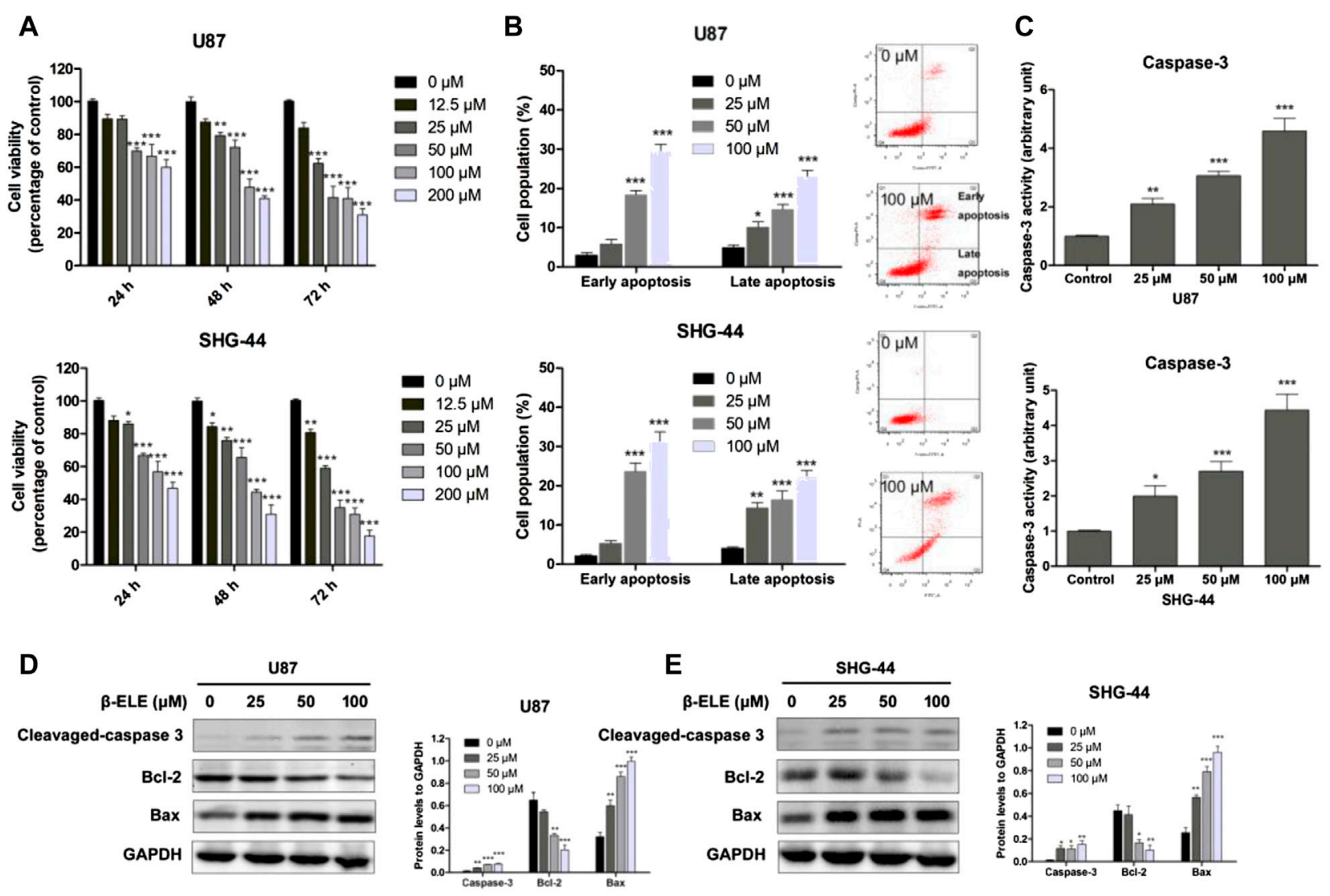

FIGURE 1 | $\beta$-Elemene decreased cell viability of glioma cells and induced cell apoptosis. (A) U87 and SHG-44 cells were treated with various concentrations $(0-200 \mu \mathrm{M})$ of $\beta$-elemene and incubated for indicated time $(24,48$ and $72 \mathrm{~h}$ ). Cell viability was determined with MTT method. (B) Cell apoptosis of glioma cells was determined by Annexin V/PI co-staining flow cytometric analysis after incubation with 0, 25, 50 and $100 \mu \mathrm{M} \beta$-elemene for $24 \mathrm{~h}$. (C) Caspase-3 activity was determined after incubation with 0, 25, 50 and $100 \mu \mathrm{M} \beta$-elemene for $12 \mathrm{~h}$ (D,E) Expression levels of cleaved-caspase 3, Bcl-2, and Bax in U87 and SHG-44 cells after treatment with $0,25,50$ and $100 \mu \mathrm{M} \beta$-elemene for $24 \mathrm{~h}$. Data are presented as mean $\pm \mathrm{SD}$ of three independent experiments, ${ }^{*} p<0.05,{ }^{* *} p<0.01,{ }^{* * *} p<0.001$, compared with control group.

\section{Statistical Analysis}

All data were expressed as means \pm SD of three replicates. Statistical comparisons were performed by Student's $t$-test to compare two groups. To compare more than two groups, oneway ANOVA with Tukey post hoc analysis was performed. Statistical significance was defined as $p<0.05$ between different groups, the analysis were performed using GraphPad Prism 5.0 software.

\section{RESULTS}

\section{$\beta$-Elemene Inhibited the Growth and Induced Apoptosis of Glioma Cells}

To verify the anticancer activity of $\beta$-elemene, the cell viabilities of U87 and SHG-44 cells were determined with MTT assay after incubation with various concentrations of $\beta$-elemene for 24,48 , and $72 \mathrm{~h}$. It was found that $\beta$-elemene induced a dose- and time-dependent inhibition of the growth of glioma cells (Figure 1A). To determine whether $\beta$-elemene induced apoptosis of these cells, Annexin V/PI co-staining was performed and the cells at early and late stages were analyzed with flow cytomery. The results showed that $\beta$-elemene induced the apoptosis of glioma cells in a dosedependent manner (Figure 1B). In addition, increased activity of caspase-3 in both U87 and SHG-44 cells after treatment with $\beta$-elemene was also observed (Figure 1C). The expression levels of cleaved caspase-3, Bcl-2, and Bax were also examined using western blot analysis in both U87 and SHG-44 cells, and it was found that $\beta$-elemene increased the expression of cleaved caspase- 3 and Bax, while decreased the expression of Bcl-2 (Figures 1D,E).

\section{$\beta$-Elemene Induced ROS Generation in Glioma Cells}

DCF-DA, a common oxidative stress indicator, was used to measure ROS in glioma cells [16]. As shown in Figure 2A, after incubation with $100 \mu \mathrm{M} \quad \beta$-elemene for $12 \mathrm{~h}$, green fluorescence was observed under fluorescence microscopy in 


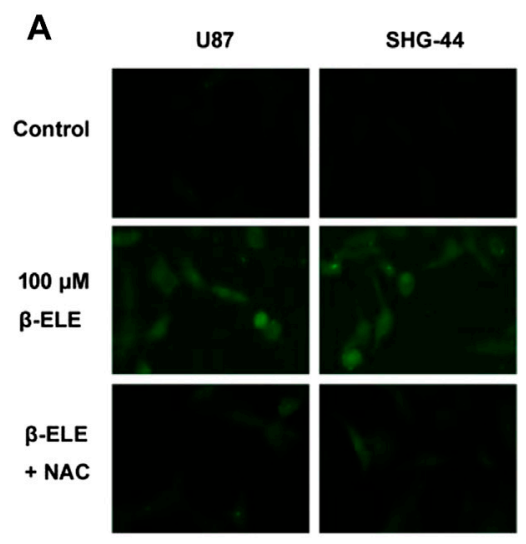

B
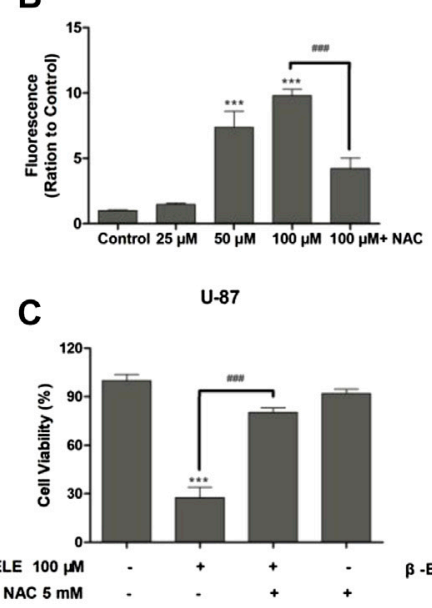

U87

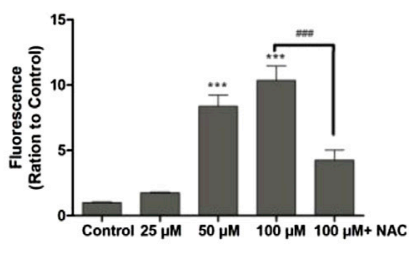

SHG-44

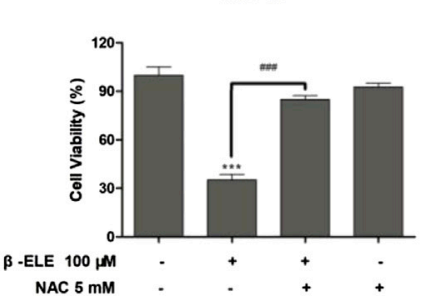

FIGURE 2 | $\beta$-Elemene increased ROS production in glioma cells. (A) The levels of ROS in U87 and SHG-44 cells were indicated by DCF-DA staining, and green fluorescence intensity indicated the degree of ROS. (B) The levels of ROS in glioma cells after treatment with 0, 25, 50 and $100 \mu \mathrm{M} \beta$-elemene for $12 \mathrm{~h}$ were measured by flow cytometric analysis, pre-incubation of cells with $5 \mathrm{mM} \mathrm{NAC}$ for $0.5 \mathrm{~h}$ reduced the production of ROS. (C) The clearance of ROS partly increased cell viability. Cell viability was analyzed by the MTT assay when U87 or SHG-44 cells were treated with $100 \mu \mathrm{M} \beta$-elemene for $48 \mathrm{~h}$ or pre-incubated with 5 mM NAC for 0.5 h. Data are presented as mean $\pm \mathrm{SD}$ of three independent experiments, ${ }^{\star \star \star} p<0.001$, compared with control group, ${ }^{\# \# \#} p<0.001$, compared with NAC treated group.

A

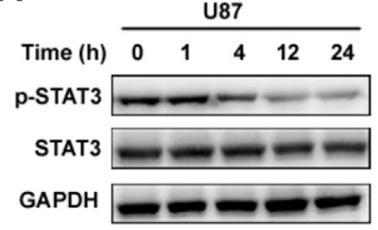

U87

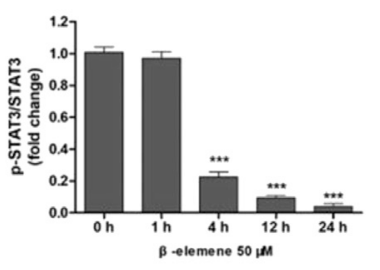

B

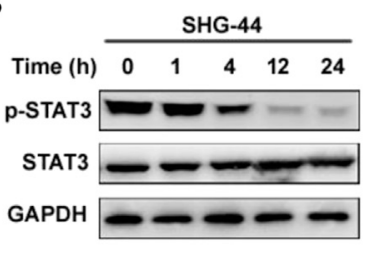

SHG-44

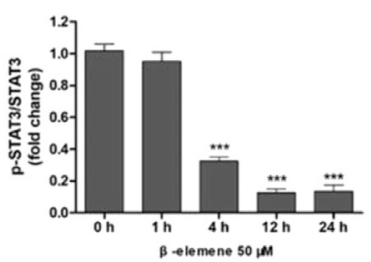

C

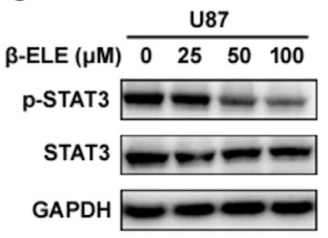

U87

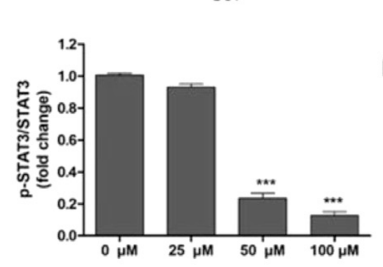

D

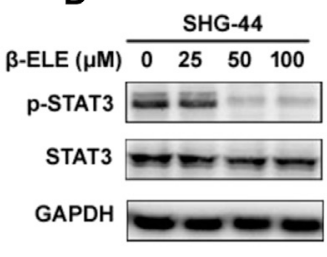

SHG-44

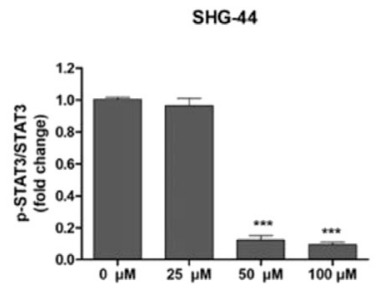

FIGURE 3 | $\beta$-Elemene suppressed phosphorylation of STAT3 in glioma cells. (A,B) Western blotting showed that $\beta$-elemene $(50 \mu \mathrm{M})$ reduced the phosphorylation of STAT3 in a time-dependent manner. The GAPDH was used as a control and the blots were quantitatively evaluated. (C,D) Western blotting showed that after treatment with $\beta$-elemene for 12 h reduced the phosphorylation of STAT3 in a dose-dependent manner. The bands corresponding to each protein were quantified using GAPDH as a control and normalized relative to band intensities in control group. Data are presented as mean \pm SD of three independent experiments, ${ }^{\star \star \star} p<0.001$, compared with control group.

both U87 and SHG-44 cells, indicating the generation of ROS. However, pre-incubation with antioxidant NAC significantly attenuated the generation of ROS in these cells. When ROS production was detected with different doses of $\beta$-elemene by analysis of fluorescence intensity, it was found that $\beta$-elemene increased the generation of ROS in a dose-dependent manner in glioma cells Figure 2B. In addition, pre-incubation of NAC not only abolished the generation of ROS, but also the cytotoxicity of $\beta$-elemene (Figure $2 \mathrm{C}$ ), confirming the critical role of ROS in $\beta$-elemene induced apoptosis.

\section{$\beta$-Elemene Suppressed Phosphorylation of STAT3 in Glioma Cells}

To verify whether $\beta$-elemene affected the expression of STAT3, U87 and SHG-44 cells were incubated with $50 \mu \mathrm{M} \beta$-elemene for 

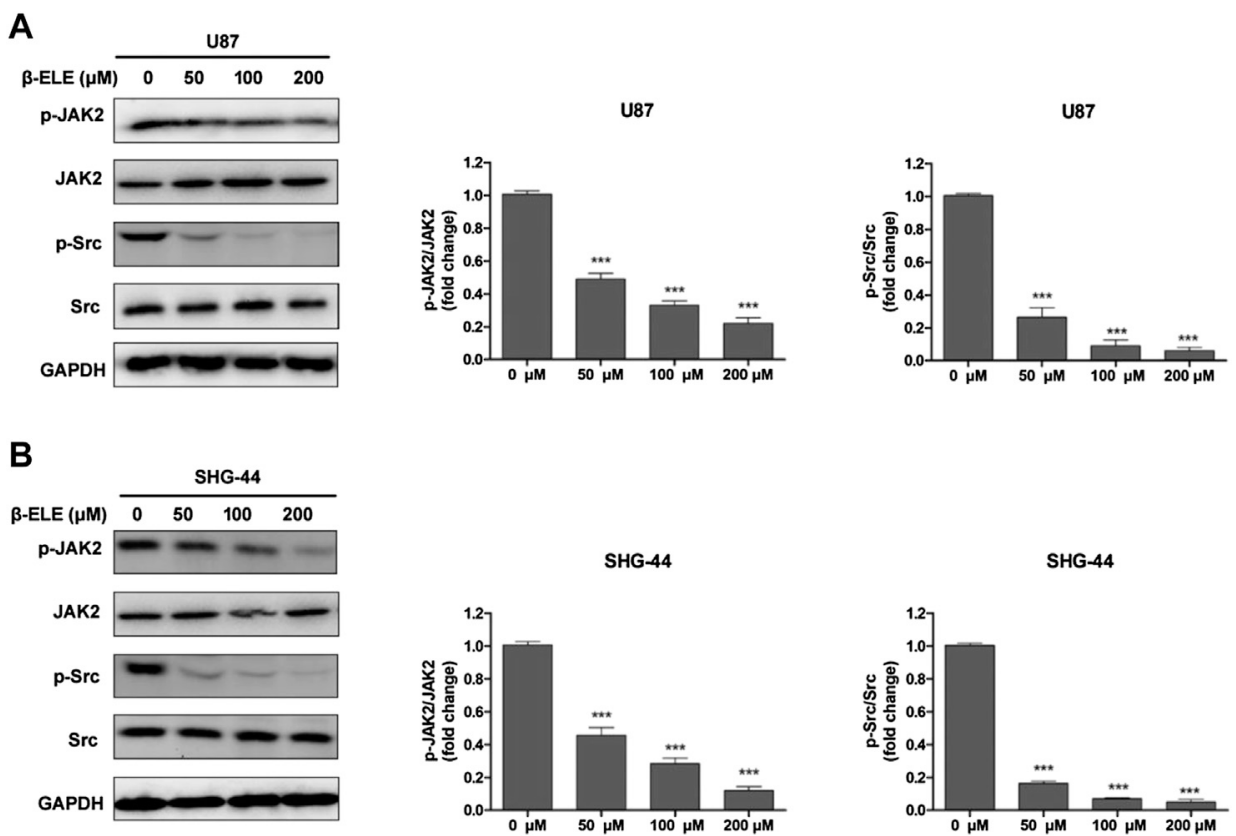

FIGURE $4 \mid \beta$-Elemene suppressed phosphorylation of JAK2 and Src in glioma cells. (A) Western blotting showed that $\beta$-elemene reduced the phosphorylation of JAK2 and Src in a dose-dependent manner in U87 cells and (B) SHG-44 cells. The bands corresponding to each protein were quantified using GAPDH as a control and normalized relative to band intensities in control group. Data are presented as mean \pm SD of three independent experiments, ${ }^{\star \star \star} p<0.001$, compared with control group.

different time and the expression levels of STAT3 and phosphoSTAT3 were detected by western blot. It was found that the expression level of p-STAT3 was decreased over time and STAT3 was not affected by $\beta$-elemene in either U87 or SHG-44 cells (Figures 3A,B). In addition, after incubation with 25, 50 and $100 \mu \mathrm{M} \beta$-elemene for $12 \mathrm{~h}$, statistical difference of p-STAT3 expression was observed in 50 and $100 \mu \mathrm{M}$ groups compared with control group (Figures 3C,D). These findings suggested that $\beta$-elemene suppressed the STAT3 activation in glioblastoma.

\section{$\beta$-Elemene Suppressed Phosphorylation of STAT3 via JAK2/Src Pathway}

It has been reported that STAT3 can be activated by Janusactivated kinases (JAKs) and c-Src [17], thus, we evaluated the expression levels of JAK2 and Src in glioma cells after incubation with $\beta$-elemene by western blot analysis. The results showed that $\beta$-elemene dose-dependently decreased the phosphorylation of JAK2 and Src in both U87 and SHG-44 cells, while the expression levels of JAK2 and Src were not affected (Figures 4A,B).

\section{STAT3 Activation Is Mediated by ROS Production in Glioma Cells}

In order to identify the association between ROS production and STAT3 activation, U87 and SHG-44 cells were pre-treated with NAC and the expression levels of STAT3 and p-STAT3 were detected. It was found that pre-incubation of NAC significantly abolished the $\beta$-elemene induced decrease of expression level of p-STAT in both U87 and SHG-44 cells, while the expression level of STAT3 was not affected (Figures 5A,B). It is reported that when cancer-related inflammatory cytokine interleukin (IL)-6 binds to its receptor, STAT3 would be activated [18]. Thus, we further tested whether the activation of STAT3 by IL- 6 would be influenced by $\beta$-elemene. It was found that exposure of glioma cells to IL-6 $(50 \mathrm{ng} / \mathrm{ml})$ increased the expression of p-STAT3, while co-incubation of $\beta$-elemene abolished such activation. However, NAC pre-incubation dramatically attenuated the activity of $\beta$-elemene to suppress IL-6-induced STAT3 activation (Figures 5C,D). In addition, $\beta$-elemene induced decline of expression levels of $p$-JAK2 and $\mathrm{p}$-Src was also reversed by NAC pre-incubation in both U87 and SHG-44 cells (Figures 5E,F). Overall, these results indicated that $\beta$-elemene suppressed the activation of STAT3 pathway through elevated ROS production in glioma cells.

\section{DISCUSSION}

Current therapeutic regimens for GBM are mainly based on surgery, chemotherapy and radiotherapy. Bioactive phytochemicals are attracting increasing attention for the prevention and treatment of GBM due to their low toxicity and unique mechanism of actions. Many natural products have been reported to exhibit potent antitumor activities against GBM, such as curcumin [19], $\beta$-elemene [20], quercetin [21], and phloretin [22]. $\beta$-Elemene has been widely reported to have potent antitumor activities in various cancer cell lines. Particularly, $\beta$-elemene exhibited potent in vitro and in vivo antitumor effects in glioma cells 

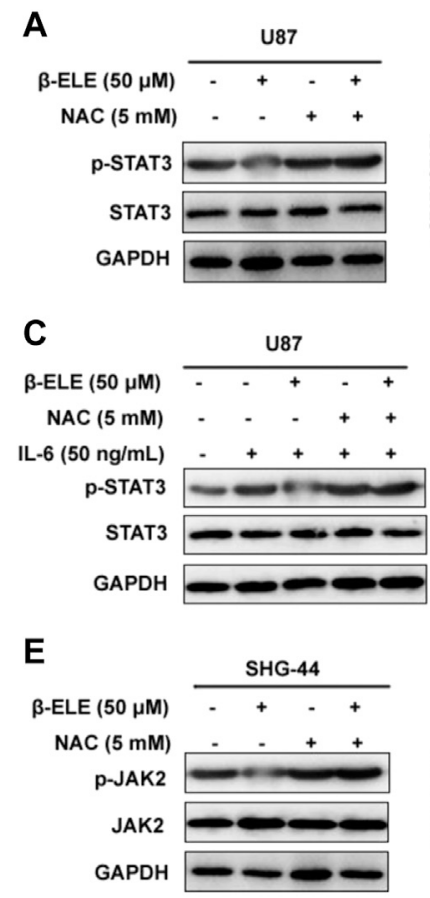

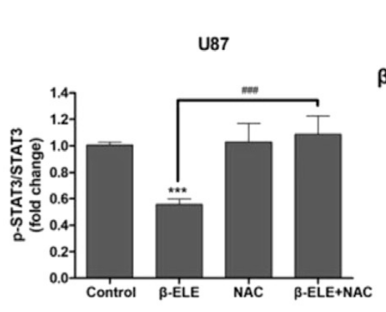

U87
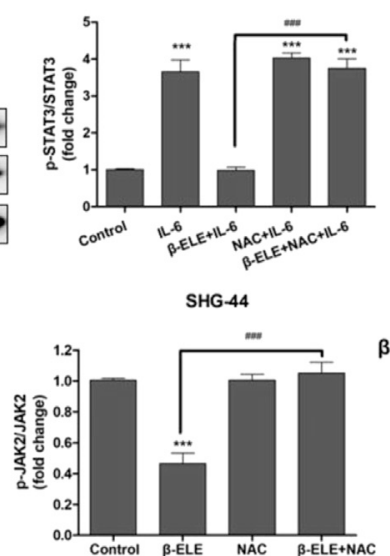
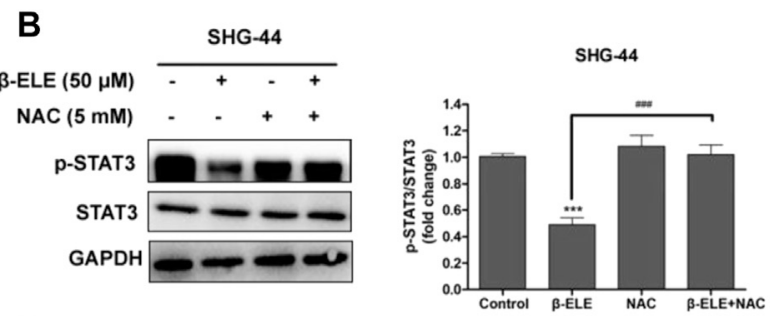

D
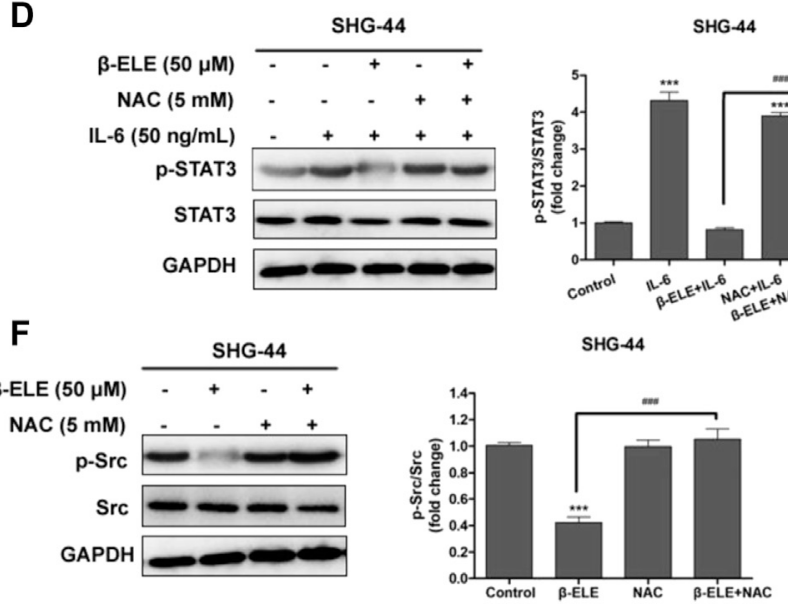

FIGURE 5 | ROS has a great effect on $\beta$-elemene-induced suppression of STAT3 signaling in glioma cells. (A,B) Cells were treated with $50 \mu \mathrm{M} \beta$-elemene in the absence or presence of $5 \mathrm{mM} \mathrm{NAC}$ for $24 \mathrm{~h}$, and cell lysates were subjected to western blot to analyze the expression of p-STAT3 and STAT3. (C,D) Cells were pretreated with $5 \mathrm{mM} \mathrm{NAC}$, and then IL-6 and $\beta$-elemene were added and incubated for $24 \mathrm{~h}$. Cell lysates were subjected to western blot to analyze the expression of p-STAT3 and STAT3. (E,F) SHG-44 cells were treated with $50 \mu \mathrm{M} \beta$-elemene in the absence or presence of $5 \mathrm{mM} \mathrm{NAC}$ for $24 \mathrm{~h}$, and cell lysates were subjected to western blot to analyze the expression of p-JAK2, JAK2, p-Src, and Src. GAPDH was used as a control and the blots were quantitatively evaluated. Data are presented as mean \pm SD of three independent experiments, ${ }^{\star \star *} p<0.001$, compared with control group, ${ }^{\# \# ~} p<0.001$, compared with NAC treated group.

[23]. In China, $\beta$-elemene has been used in clinical practice as an adjuvant medicine in the treatment of GBM. However, the detailed antitumor mechanism of $\beta$-elemene in glioma cells remains unclear. Illumination of its mechanism would accelerate its further development as an antitumor agent for the treatment of GBM.

Apoptosis is a specific and programmed mechanism of cell death which regulates homeostasis of tissues and elimination of malignant cells. The apoptotic process is involved in the mechanism of many natural products, including $\beta$-elemene. In this study, we found that $\beta$-elemene decreased cell viability and induced cell apoptosis of U87 and SHG-44 glioma cells. $\beta$-Elemene induced apoptosis in glioma cells was associated with the activation and cleavage of caspase- 3 . Jiang and co-workers also reported that $\beta$-elemene induced apoptosis in human glioma cells by regulation of Fas/FasL and activation of caspase-3, -8 and -9 [24]. Shi and coworkers showed that $\beta$-elemene induced glioma cell apoptosis by downregulating survivin and its interaction with hepatitis $\mathrm{B}$ X-interacting protein [25].

Many factors would induce apoptosis in cancer cells, and numerous studies have illustrated that excessive accumulation of intracellular ROS is a signal for initiating apoptosis [26]. Many natural products have been reported to induce apoptosis of cancer cells through generation of ROS. For example, ouabain is reported to elicit human glioblastoma cells apoptosis by generating ROS in ERK-p66SHC-dependent pathway [27]. Huang and co-workers reported that phloretin induces cell cycle arrest and apoptosis of human glioblastoma cells through the generation of ROS [22]. However, the exact role of ROS in cells is controversial due to its action as a double-edged sword. At low levels, ROS promotes tumor development through activating signaling pathways to accelerate the proliferation and differentiation of cells, while at high levels, ROS is toxic and results in oxidative damage and cell death. Cancer cells often have increased oxidative stress compared with normal cells and are more vulnerable to ROS insults. Accumulation of excessive ROS resulted in oxidative DNA damage, mitochondrial dysfunction, and enzyme inactivation, making it an important target for development of anticancer drugs. The present study showed that $\beta$-elemene induced ROS generation in U87 and SHG-44 cells in a dose- and time-dependent manner. In addition, $\beta$-elemene induced ROS generation could be blocked by an antioxidant, NAC. Similarly, the apoptotic activity of $\beta$-elemene is also ROS-dependent, pre-incubation of U87 and SHG-44 cells with NAC abolished its apoptotic activity. These results suggested that ROS generation is critical in $\beta$-elemene induced antitumor activity in GBM cells. 
STAT3 is a cytosolic transcription factor which regulates angiogenesis, proliferation and survival of cells. Constitutive activation or aberrant phosphorylation of STAT3 are often identified in many human cancer cells, including breast lung, prostate and glioblastoma [28]. Phosphorylated STAT3 is dimerized and transferred into the nucleus to further regulate genes involved in cell survival, proliferation, immune regulation and invasion. Targeting STAT3 signaling by small molecules might be a potential strategy for the treatment of human cancer. In this study, we found that $\beta$-elemene inhibited the growth of glioblastoma cells and induced apoptosis, accompanied by remarkable suppression of active STAT3 in a dose- and time-dependent manner. In addition, we further explored the association between ROS generation and STAT3 activation. The results showed that $\beta$-elemene induced STAT3 activation was blocked by an antioxidant, NAC, which is a ROS inhibitor, suggesting that $\beta$-elemene abrogated STAT3 activation via ROS-mediated oxidative damage and then induced apoptosis in glioblastoma cells.

Overall, our data suggest that $\beta$-elemene can inhibit the growth of glioblastoma cells through suppressing STAT3 signaling pathway, which is mainly regulated by ROSmediated oxidative stress. Our study implied that $\beta$-elemene could be developed as a potential antitumor agent for the treatment of GBM through targeting STAT3.

\section{REFERENCES}

1. Preusser M, de Ribaupierre S, Wöhrer A, Erridge SC, Hegi M, Weller M, et al. Current concepts and management of glioblastoma. Ann Neurol. (2011). 70(1): 9-21. doi:10.1002/ana.22425

2. Hombach-Klonisch S, Mehrpour M, Shojaei S, Harlos C, Pitz M, Hamai A, et al. Glioblastoma and chemoresistance to alkylating agents: involvement of apoptosis, autophagy, and unfolded protein response. Pharmacology Ther (2018). 184:13-41. doi:10.1016/j.pharmthera.2017.10.017

3. Osuka S, Van Meir EG Overcoming therapeutic resistance in glioblastoma: the way forward. J Clin Invest (2017). 127(2):415-26. doi:10.1172/jci89587

4. Geraldo LHM, Garcia C, da Fonseca ACC, Dubois LGF, de Sampaio e Spohr TCL, Matias D, et al. Glioblastoma therapy in the age of molecular medicine. Trends Cancer (2019). 5(1):46-65. doi:10.1016/j.trecan.2018.11.002

5. McGranahan T, Therkelsen KE, Ahmad S, Nagpal S Current state of immunotherapy for treatment of glioblastoma. Curr Treat Options Oncol (2019). 20(3):24. doi:10.1007/s11864-019-0619-4

6. Liu Y, Li C, Lin J STAT3 as a therapeutic target for glioblastoma. Acamc (2010). 10(7):512-9. doi:10.2174/187152010793498636

7. Furtek SL, Backos DS, Matheson CJ, Reigan P Strategies and approaches of targeting STAT3 for cancer treatment. ACS Chem. Biol. (2016). 11(2):308-18. doi:10.1021/acschembio.5b00945

8. Kang S-H, Yu MO, Park K-J, Chi S-G, Park D-H, Chung Y-G Activated STAT3 regulates hypoxia-induced angiogenesis and cell migration in human glioblastoma. Neurosurgery (2010). 67(5):1386-95; discussion 1395. doi:10. 1227/neu.0b013e3181f1c0cd

9. Jackson C, Ruzevick J, Amin AG, Lim M Potential role for STAT3 inhibitors in glioblastoma. Neurosurg Clin North America (2012). 23(3):379-89. doi:10.1016/ j.nec.2012.04.002

10. Zhai B, Zhang N, Han X, Li Q, Zhang M, Chen X, et al. Molecular targets of $\beta$-elemene, a herbal extract used in traditional Chinese medicine, and its potential role in cancer therapy: a review. Biomed Pharmacother (2019). 114: 108812. doi:10.1016/j.biopha.2019.108812

\section{DATA AVAILABILITY STATEMENT}

The original contributions presented in the study are included in the article/Supplementary Material, further inquiries can be directed to the corresponding authors.

\section{AUTHOR CONTRIBUTIONS}

SC and QX contributed equally to this work.

\section{FUNDING}

This publication was made possible by research supported by the Project of Invigorating Healthcare through Science, Technology and Education (No. kjxw2018020 to Y-t Ji), the National Natural Science Foundation of China (No. 81702065 to L-n Zhao), and the National Natural Science Foundation of Jiangsu province (No. BK20170364)

\section{CONFLICT OF INTEREST}

The authors declare that the research was conducted in the absence of any commercial or financial relationships that could be construed as a potential conflict of interest.

11. Qureshi MZ, Attar R, Romero MA, Sabitaliyevich UY, Nurmurzayevich SB, Ozturk $\mathrm{O}$, et al. Regulation of signaling pathways by $\beta$-elemene in cancer progression and metastasis. J Cel Biochem (2019). 120(8):12091-100. doi:10. $1002 /$ jcb. 28624

12. Guan C, Liu W, Yue Y, Jin H, Wang X, Wang XJ Inhibitory effect of $\beta$-elemene on human breast cancer cells. Int J Clin Exp Pathol (2014). 7(7):3948-56.

13. Yu X, Xu M, Li N, Li Z, Li H, Shao S, et al. $\beta$-elemene inhibits tumor-promoting effect of M2 macrophages in lung cancer. Biochem Biophysical Res Commun (2017). 490(2):514-20. doi:10.1016/j.bbrc.2017.06.071

14. Wu XS, Xie T, Lin J, Fan HZ, Huang-Fu HJ, Ni LF, et al. An investigation of the ability of elemene to pass through the blood-brain barrier and its effect on brain carcinomas. J Pharm Pharmacol (2009). 61(12):1653-6. doi:10.1211/jpp/ 61.12.0010

15. Chen J, Wang T, Xu S, Lin A, Yao H, Xie W, et al. Novel hybrids of natural $\beta$ -elemene bearing isopropanolamine moieties: synthesis, enhanced anticancer profile, and improved aqueous solubility. Fitoterapia (2017). 120:117-25. doi:10.1016/j.fitote.2017.05.002

16. Boulton S, Anderson A, Swalwell H, Henderson JR, Manning P, Birch-Machin MA Implications of using the fluorescent probes, dihydrorhodamine 123 and 2',7'-dichlorodihydrofluorecein diacetate, for the detection of UVA-induced reactive oxygen species. Free Radic Res (2011). 45(2):139-46. doi:10.3109/ 10715762.2010.517751

17. Slattery ML, Lundgreen A, Kadlubar SA, Bondurant KL, Wolff RK JAK/STAT/ SOCS-signaling pathway and colon and rectal cancer. Mol. Carcinog. (2013). 52(2):155-66. doi:10.1002/mc.21841

18. Mitsuyama K, Matsumoto S, Masuda J, Yamasakii H, Kuwaki K, Takedatsu H, et al. Therapeutic strategies for targeting the IL-6/STAT3 cytokine signaling pathway in inflammatory bowel disease. Anticancer Res (2007). 27(6A):3749-56.

19. Shabaninejad Z, Pourhanifeh MH, Movahedpour A, Mottaghi R, Nickdasti A, Mortezapour E, et al. Therapeutic potentials of curcumin in the treatment of glioblstoma. Eur J Med Chem (2020). 188:112040. doi:10.1016/j.ejmech.2020. 112040

20. Chen J, Wang T, Xu S, Zhang P, Lin A, Wu L, et al. Discovery of novel antitumor nitric oxide-donating $\beta$-elemene hybrids through inhibiting the 
PI3K/Akt pathway. Eur J Med Chem (2017). 135:414-23. doi:10.1016/j.ejmech. 2017.04.045

21. Tavana E, Mollazadeh H, Mohtashami E, Modaresi SMS, Hosseini A, Sabri H, et al. Quercetin: A promising phytochemical for the treatment of glioblastoma multiforme. Biofactors (2020). 46:356-66. doi:10.1002/biof.1605

22. Liu Y, Fan C, Pu L, Wei C, Jin H, Teng Y, et al. Phloretin induces cell cycle arrest and apoptosis of human glioblastoma cells through the generation of reactive oxygen species. J Neurooncol (2016). 128(2):217-23. doi:10.1007/s11060-016-2107-z

23. Zhu Y, Hu J, Shen F, Shen H, Liu W, Zhang J The cytotoxic effect of $\beta$-elemene against malignant glioma is enhanced by base-excision repair inhibitor methoxyamine. J Neurooncol (2013). 113(3):375-84. doi:10.1007/s11060013-1136-0

24. Li CL, Chang L, Guo L, Zhao D, Liu HB, Wang QS, et al. $\beta$-elemene induces caspase-dependent apoptosis in human glioma cells in vitro through the upregulation of Bax and Fas/FasL and downregulation of Bcl-2. Asian Pac J Cancer Prev (2014). 15(23):10407-12. doi:10.7314/apjcp.2014.15.23.10407

25. Zhang $\mathrm{H}, \mathrm{Xu}$ F, Xie T, Jin H, Shi L $\beta$-elemene induces glioma cell apoptosis by downregulating survivin and its interaction with hepatitis B X-interacting protein. Oncol Rep (2012). 28(6):2083-90. doi:10.3892/or.2012.2022
26. Simon H-U, Haj-Yehia A, Levi-Schaffer F Role of reactive oxygen species (ROS) in apoptosis induction. Apoptosis (2000). 5(5):415-8. doi:10.1023/a: 1009616228304

27. Yan X, Liang F, Li D, Zheng J Ouabain elicits human glioblastoma cells apoptosis by generating reactive oxygen species in ERK-p66SHC-dependent pathway. Mol Cel Biochem (2015). 398(1-2):95-104. doi:10.1007/s11010-0142208-y

28. Balic JJ, Garama DJ, Saad MI, Yu L, West AC, West AJ, et al. Serinephosphorylated STAT3 promotes tumorigenesis via modulation of RNA polymerase transcriptional activity. Cancer Res (2019). 79(20):5272-87. doi:10.1158/0008-5472.can-19-0974

Copyright $\odot 2021$ Cai, Xiong, Zhao, Ji, Luo and Ma. This is an open-access article distributed under the terms of the Creative Commons Attribution License (CC BY). The use, distribution or reproduction in other forums is permitted, provided the original author(s) and the copyright owner(s) are credited and that the original publication in this journal is cited, in accordance with accepted academic practice. No use, distribution or reproduction is permitted which does not comply with these terms. 